\title{
Original
}

\section{Optogenetic Stimulation of 5-HT Neurons in the Median Raphe Nucleus Affects Anxiety and Respiration}

\author{
Naoki OKuma ${ }^{1)}$, Mitsuko KanAmaru*1), Rika MoriYA ${ }^{1)}$, \\ Kenji F. TANAKA ${ }^{2)}$, Satoru Arata ${ }^{3)}$, Jun WatanABE ${ }^{3)}$, \\ Akira YoshikawA $^{1)}$ and Masahiko IzUMIZAKI $^{1)}$
}

\begin{abstract}
Anxiety affects respiration, and in turn perturbs the internal environment, although the neuronal systems controlling anxiety-related respiration remain unclear. Recent reports indicate that serotonin (5-HT) neurons in the median raphe nucleus (MRN) enhance anxiety. In the present study, we aimed to clarify the contribution of 5-HT neurons in the MRN to anxiety and respiratory control using mice expressing a channelrhodopsin-2 variant-enhanced yellow fluorescent protein (ChR2 [C128S]-EYFP; a step-function opsin) in the central 5-HT neurons. We applied an optogenetic method to bigenic mice expressing ChR2 [C128S]-EYFP in 5-HT neurons and to monogenic mice without such expression. Photostimulation of freemoving mice was performed using a wireless system through an optical fiber preinserted above the MRN, and respiratory variables were measured using wholebody plethysmography. Anxiety was evaluated using an elevated-plus maze test. In the bigenic mice, we confirmed ChR2 [C128S]-EYFP expression in tryptophan hydroxylase 2 (a brain 5-HT synthase)-positive neurons in the raphe nuclei of the mesopontine, such as the MRN and the dorsal raphe nucleus. Blue light illumination to the MRN of the bigenic mice significantly increased respiratory rate and minute ventilation without affecting tidal volume, and significantly decreased the time spent in the open arms of the elevated plus maze without changing distance traveled, compared with monogenic mice. These results suggest that 5-HT neuron activity in the MRN increases anxiety-like behavior without affecting locomotor activity, enhances respiratory rhythm and minute ventilation without changing tidal volume, and can mimic anxiety-related respiratory responses in humans.
\end{abstract}

Key words : optogenetics, serotonin, mice, respiration, anxiety

\section{Introduction}

Anxiety and related symptoms are frequently accompanied by respiratory dysregulation, such as sighs, episodic rapid rates, and apneas ${ }^{1-3)}$. The stressful situation itself can induce rapid deep breathing, while tense, anticipatory events can be associated with rapid shallow breathing ${ }^{4)}$.

\footnotetext{
${ }^{1)}$ Department of Physiology, Showa University School of Medicine, 1-5-8 Hatanodai, Shinagawa-ku, Tokyo 142-8555, Japan.

2) Department of Neuropsychiatry, Keio University School of Medicine.

3) Center for Biotechnology, Showa University School of Medicine.

* To whom corresponding should be addressed.
} 
Generally, breathing plays an important role in maintaining homeostasis in the body. Respiratory control based on metabolism is mainly integrated in the medulla oblongata; however, the neuronal mediators and brain regions involved in emotional control, such as anxiety-related respiratory control, are less well understood.

Central serotonin (5-HT) neurons are involved in a wide range of functions, including feeding, neuroendocrine activity, aggression, anxiety, depression ${ }^{5)}$, and the metabolic control of respiration mediated via the medulla oblongata ${ }^{6}$. Selective serotonin reuptake inhibitors are widely used for anxiety disorders, occasionally leading to an initial increase in anxiety ${ }^{7)}$. 5-HT neurons in the median raphe nucleus (MRN), related to anxiogenic behaviors ${ }^{8,9)}$, have a high density of fibers that project from the MRN to the hippocampus ${ }^{10)}$, and the septo-hippocampal axis is a crossroad between the respiratory and limbic pathways ${ }^{11)}$. Therefore, 5-HT neurons in the MRN may be candidate neurons for anxiety-related respiratory control.

Optogenetics is a powerful tool to explore the basic neuronal and circuit dynamics of fundamental physiology, higher cognitive function, and emotional behaviors in freely moving mice ${ }^{12,13)}$. It uses ChR2, which is a light-switched cation-selective ion channel, to generate a large permeability for monovalent and divalent cations ${ }^{14)}$. A modified ChR2 (ChR2 [C128S]) extends the lifetime of the open state to at least $1 \mathrm{~min}$ with blue light illumination, depolarizing cells, while yellow light illumination closes the light-switched cation-selective ion channel, terminating the depolarization of cells ${ }^{15)}$. ChR2 [C128S]-EYFP are highly expressed in cells with the improved tetracyclinecontrolled gene induction ${ }^{16)}$. Therefore, through ChR2 [C128S]-EYFP expression in central 5-HT neurons of mice, blue light illumination efficiently depolarizes 5-HT neurons for at least $1 \mathrm{~min}$, while the following yellow light illumination terminates 5 -HT neuronal depolarization ${ }^{17}$.

In this study, we thus used genetically modified mice expressing a light-sensitive ion channel in 5-HT neurons of the brain, and optogenetically studied the roles of MRN 5-HT neurons in anxiety and respiration using whole body plethysmography and an elevated plus maze test.

\section{Materials and methods}

Mice

We generated tetracycline operator channelrhodopsin-2 variant-enhanced yellow fluorescent protein (tetO-ChR2 [C128S]-EYFP; a step-function opsin) knock-in mice ${ }^{16)}$, and then crossed them with tryptophan hydroxylase 2-tetracycline-controlled transcriptional activator (Tph2-tTA) BAC transgenic mice. RIKEN BRC (Tsukuba, Japan) provided both lines through the National Bio-Resource Project of the MEXT, Japan. The experimental mice were Tph2-tTA::tetO-ChR2 [C128S]-EYFP bigenic mice, and the control mice were the monogenic, ChR2 [C128S]-EYFP negative littermates. Male mice were used and had free access to food and water except during experimental periods. Animals were housed under a 12/12-h light/dark cycle with lights on at 8:00 AM. Room temperature was approximately $26^{\circ} \mathrm{C}$. Experimental protocols were approved by the Institutional Animal Care and Use Committee of Showa University (Permit Numbers 05044 and 06090), which operates in accordance with the Japanese Government guidelines for the care and use of laboratory animals. 


\section{Histology}

Mice were intraperitoneally anesthetized with medetomidine/midazolam/butorphanol (0.3/4/5 mg/10 ml/kg body weight), perfused with saline followed by $4 \%$ paraformaldehyde, and then the brains were removed. For direct fluorescence detection, a coronal section cut at -2.6 to $-4.6 \mathrm{~mm}$ from the bregma was prepared and cleared using the advanced Clear, Unobstructed Brain / Body Imaging Cocktail (CUBIC) ${ }^{18)}$. Reagent-1 was prepared as a mixture of 25 wt\% urea (Nacalai Tesque Inc., Kyoto, Japan), 25 wt\% N,N,N',N'-tetrakis (2-hydroxypropyl) ethylenediamine (Tokyo Chemical Industry Co., LTD., Tokyo, Japan), and 15 wt\% polyethylene glycol mono-p-isooctylphenyl ether/Triton X-100 (Wako Pure Chemical Industries Ltd., Osaka, Japan). Reagent-2 was prepared as a mixture of $50 \mathrm{wt} \%$ sucrose (Wako Pure Chemical Industries, Ltd.), $25 \mathrm{wt} \%$ urea, and $10 \mathrm{wt} \%$ 2, 20, 20'-nitrilotriethanol (Wako Pure Chemical Industries, Ltd.). The brain was immersed in $1 / 2$-water-diluted reagent- 1 with rotation at $37^{\circ} \mathrm{C}$ for 3-6 h, followed by reagent- 1 alone with rotation at $37^{\circ} \mathrm{C}$ overnight. Reagent- 1 was replaced every 2 days. After clearing was complete, the brain was washed three times in phosphatebuffered saline (PBS) with rotation at room temperature for at least $2 \mathrm{~h}$ per wash, and then washed in PBS overnight with rotation at room temperature. The brain was then immersed overnight in $1 / 2$-PBS-diluted reagent- 2 with rotation at $37^{\circ} \mathrm{C}$, followed by reagent-2 alone with rotation at $37^{\circ} \mathrm{C}$ overnight. Reagent-2 was replaced with fresh reagent and further incubated for $\sim 24 \mathrm{~h}$. The cleared section was imaged by fluorescence microscopy, using $535 \mathrm{~nm} \pm 15 \mathrm{~nm}$ emission and $500 \mathrm{~nm} \pm 10 \mathrm{~nm}$ excitation (BZ-X700; Keyence, Osaka, Japan; EYFP expression is shown in yellow).

For immunohistochemistry, the other fixed brains were frozen and cut into $30-\mu \mathrm{m}$ coronal sections. After rinsing in 0.01 M PBS with 0.1\% TritonX-100 (PBST), sections were immersed in 5\% normal horse serum (Vec S-2000 Vector Laboratories, Burlingame, CA, USA) in PBST for $1 \mathrm{~h}$ at room temperature to block non-specific reactions. Sections were then incubated with the primary antibodies, mouse anti-Tph2 (T0678, 1:2000; Sigma Aldrich Japan, Tokyo) and rabbit anti-GFP (A6455, 1:1000; Life Technology Japan, Tokyo) in 5\% normal horse serum with PBST for $48 \mathrm{~h}$ at $4^{\circ} \mathrm{C}$. After rinsing several times with PBST, the sections were incubated with the secondary antibodies, Alexa Fluor 546 goat anti-mouse (1:200; Invitrogen, Eugene, OR, USA) and Alexa Fluor 488 goat anti-rabbit (1:200; Invitrogen) for $2 \mathrm{~h}$ at room temperature. After rinsing several times with $0.01 \mathrm{M}$ PBS, the samples were stained with 4,6-diamidino-2phenylindole, dihydrochloride (DAPI) (Life Technologies, Eugene, OR, USA) and mounted with antifade reagent. The stained slices were observed using a confocal scanning laser microscope (FV1000D IX81, Olympus Corporation, Tokyo, Japan).

\section{Surgical procedures}

Male mice weighing $29.6 \pm 0.6 \mathrm{~g}$ were intraperitoneally anesthetized using the combination anesthetic, and then fixed to a stereotaxic apparatus in the prone position. A head skin incision was dorsally performed with local anesthetic, bupivacaine hydrochloride hydrate, and the bone was drilled. A bundle of two optical fibers with blue and yellow light-emitting diodes (LEDs) 
(TeleLCT, Bioresearch Center, Nagoya, Japan) was inserted above the MRN (4.5 mm posterior to the bregma, $2.5 \mathrm{~mm}$ lateral to the midline, and $3.8 \mathrm{~mm}$ deep from the dorsal brain surface, at a $35^{\circ}$ angle to an axial bar), and then fixed onto the skull with dental cement.

\section{Photostimulation}

Following at least a 1-week recovery after the optical fiber insertion, photostimulation was performed using a stimulator and a wireless receiver. The mice were intermittently stimulated with blue light $(0.25 \mathrm{~mm}$ diameter, $8 \mathrm{~mW}$ ) for $1 \mathrm{~min}$ (each exposure $500 \mathrm{~ms}$ ) and similarly with yellow light $(0.25 \mathrm{~mm}$ diameter, $8 \mathrm{~mW})$. Then, the photostimulation was repeated 5 times in the same pattern. The location of the optical fiber was evaluated on $50-\mu \mathrm{m}$ coronal sections using light microscopy.

\section{Respiratory experiment}

The whole-body plethysmograph chamber was continuously flushed with room air, connected to a differential pressure sensor, and then calibrated to air injected from a 1-ml syringe. The flow signal was acquired at $1 \mathrm{kHz}$ with continuous correction of chamber temperature and humidity. Based on the barometric plethysmography, respiratory rate (RR), inspiratory time (TI), expiratory time (TE), tidal volume (VT), and minute ventilation (MV) were measured (PLT-UNR-MR-2; Emka Japan, Kobe, Japan). The room lights were turned off $(<5$ lux $)$ and mice pre-inserted with the optical fiber were acclimatized for $90 \mathrm{~min}$ in a dark chamber to make them active even during the light period. The free-moving mice in the dark chamber were photostimulated as described above. Respiratory variables per minute during a 1-min baseline and 10-min photostimulation period were averaged and analyzed.

\section{Anxiety-like behavior experiment}

After at least 3 days of respiratory curve recording, the mice underwent the elevated plus maze test in an apparatus consisting of two open arms $(29.5 \times 5 \times 0.2 \mathrm{~cm}$ each $)$, two enclosed arms $(29.5 \times 5 \times 20 \mathrm{~cm}$ each $)$, and a central platform $(5 \times 5 \mathrm{~cm})$ elevated $41 \mathrm{~cm}$ above the floor. The two arms of each type were opposite to each other, and a wireless receiver was attached to the optical fiber pre-inserted above the MRN. The mice were acclimatized for $90 \mathrm{~min}$ in a dark room ( $<5$ lux $)$ during the light period, and then placed on a central platform facing an open arm. The mice movements during photostimulation as described above were then recorded and measured for $10 \mathrm{~min}$ using a video tracking system (Smart ver. 2.5.15, Panlab, Barcelona, Spain) to analyze the amount of time spent in the open arms and the total distance traveled.

\section{Statistics}

All data are expressed as mean \pm standard error. The time course changes expressed as line graphs between the bigenic and monogenic groups were compared by two-way repeated measures ANOVA. In cases of a significant photostimulation $\mathrm{x}$ genotype interaction, Dunnett's test was performed to determine significant differences at specific times relative to baseline values. 
SPSS Statistics ver. 22.0 (IBM Japan, Tokyo) was used in all statistical analyses. $P<0.05$ was considered statistically significant.

\section{Results}

\section{Localization of 5-HT neurons in the MRN}

A 2-mm thick coronal section (Fig. 1a) was cleared with the advanced CUBIC reagents (Fig. 1b). The EYFP fluorescence shown in yellow was distributed in the MRN and the dorsal raphe nucleus (DRN) (Fig. 1c, d). The ChR2 [C128S]-EYFP-positive cells by immunohistochemistry are shown in green (Fig. 1e, left), while Tph2-positive cells are shown in red (Fig. 1e, center). The arrowheads indicate colocalized ChR2 [C128S]-EYFP and Tph2 cells. DAPI staining of cell nuclei is shown in dark blue (Fig. 1e, right).

Respiratory responses to optogenetic stimulation of ChR2 [C128S]-EYFP-expressing 5-HT neurons in the MRN

Respiratory flow curves and respiratory variables are shown in Figure 2 and in Figures 3, 4, respectively. Each cycle of the respiratory flow curves indicates a cycle of respiration, and blue light illumination of the ChR2 [C128S]-EYFP-expressing 5-HT neurons in the MRN facilitated respiration in conscious mice, with the responses decreasing during yellow light illumination (Fig. 2). In the bigenic group, the first and second blue light illumination significantly elicited changes in RR, TI, TE, and MV compared with baseline values, but did not affect VT. The changes induced by the blue light illumination decreased during yellow light illumination. The respiratory responses in the bigenic group were significantly different from those in the monogenic group (Fig. 3), whereby in the averaged data based on the five repeated blue and yellow light illuminations, respiratory responses including $\mathrm{RR}$, TI, TE, and MV (but not VT) were significantly different from those in the monogenic group (Fig. 4). Thus, blue light illumination to the MRN increased RR by decreasing TI and TE without changing VT, and increased MV. Time courses of respiratory variables in Figure 3 are based on the same data in Figure 4, but help to understand respiratory curve responses in Figure 2.

Anxiety-like behavior elicited by optogenetic stimulation of ChR2 [C128S]-EYFP-expressing 5-HT neurons in the MRN

In the elevated plus maze test, the mean time spent on the open arms and the total distance traveled were averaged from the five repeated blue and yellow light illuminations. In the bigenic group, blue light illumination to the MRN shortened the mean time spent in the open arms, and when this was followed by illumination with yellow light, the amount of time spent in the open arms was prolonged. The change in the time spent in the open arms in the bigenic group was significantly different from that in the monogenic group (Fig. 5a). Illumination with both blue and yellow lights did not affect the total distance traveled in the bigenic and monogenic groups (Fig. 5b). 

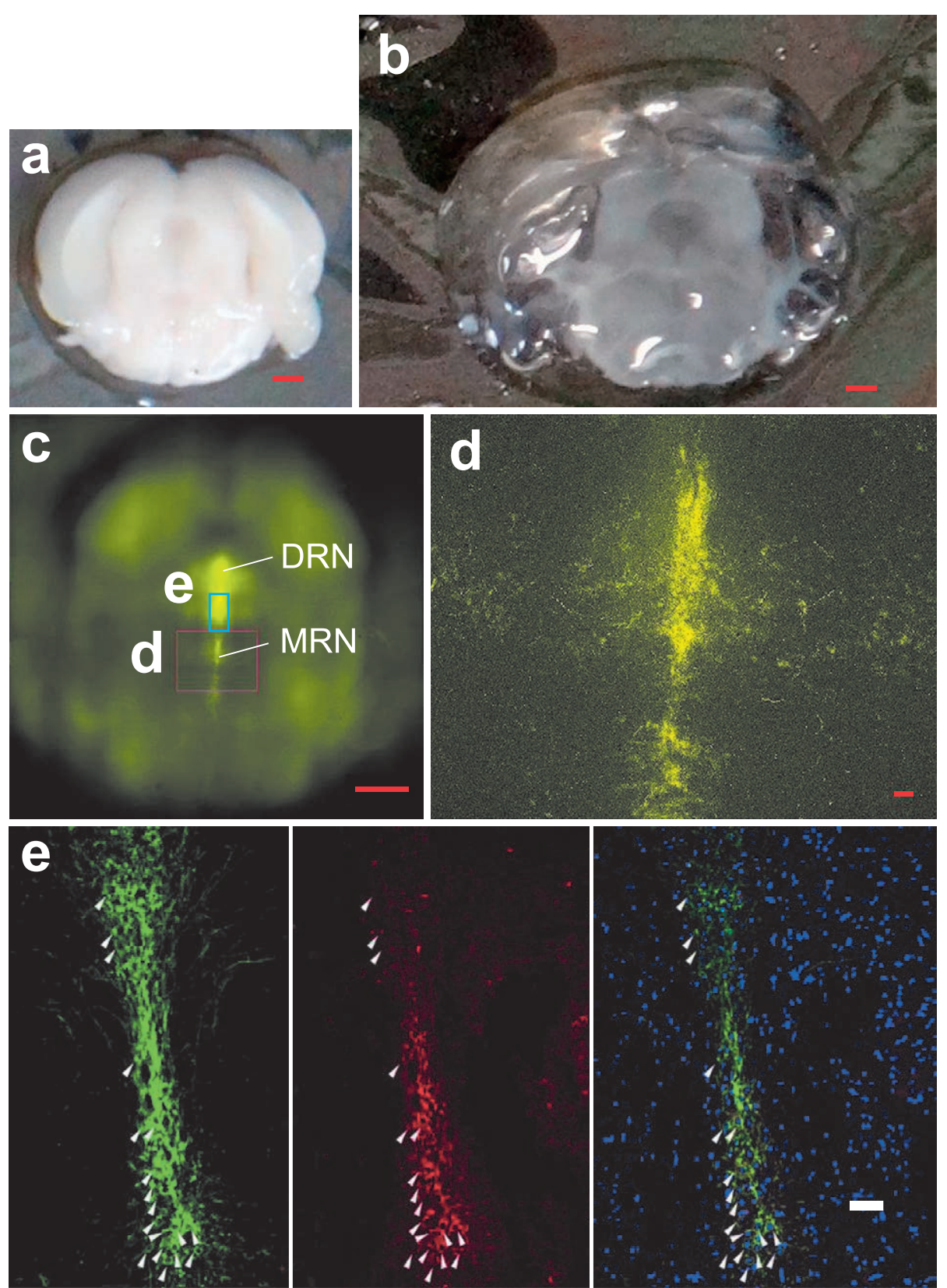

ChR2-EYFP

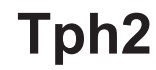

overlay

Fig. 1. Anatomical and histological considerations

a: Coronal section of the brain following $4 \%$ paraformaldehyde perfusion, at the level of -2.6 to $-4.6 \mathrm{~mm}$ from the bregma. Scale bar $=1 \mathrm{~mm}$.

$\mathrm{b}$ : Coronal section at the level of -2.6 to $-4.6 \mathrm{~mm}$ from the bregma cleared by immersion in the advanced Clear, Unobstructed Brain / Body Imaging Cocktail ${ }^{18)}$. Scale bar $=1 \mathrm{~mm}$.

$\mathrm{c}$ : Caudal-side image of $\mathrm{b}$ from a bigenic mouse [tryptophan hydroxylase 2-tetracycline-controlled transcriptional activator::tetracycline operator-channelrhodopsin-2 [C128S]-enhanced yellow fluorescent protein (EYFP) mouse], indicating the distribution of EYFP-positive neurons in the mesopontine. Scale bar $=1 \mathrm{~mm}$. DRN : dorsal raphe nucleus, MRN: median raphe nucleus.

$\mathrm{d}$ : Magnified and stacked image of the indicated area in $\mathrm{c}$ at the level -2.6 to $-4.6 \mathrm{~mm}$ from the bregma, indicating EYFP-positive neurons in MRN. Scale bar $=50 \mu \mathrm{m}$.

e: Co-localization of ChR2 [C128S]-EYFP and Tph2 in cell bodies of the raphe nuclei of the mesopontine. Scale bar $=50 \mu \mathrm{m}$. Arrowheads indicate immunohistochemical-positive cells for both ChR2 [C128S]-EYFP and Tph2, demonstrating localization of ChR2 [C128S]-EYFP in 5-HT neurons. 


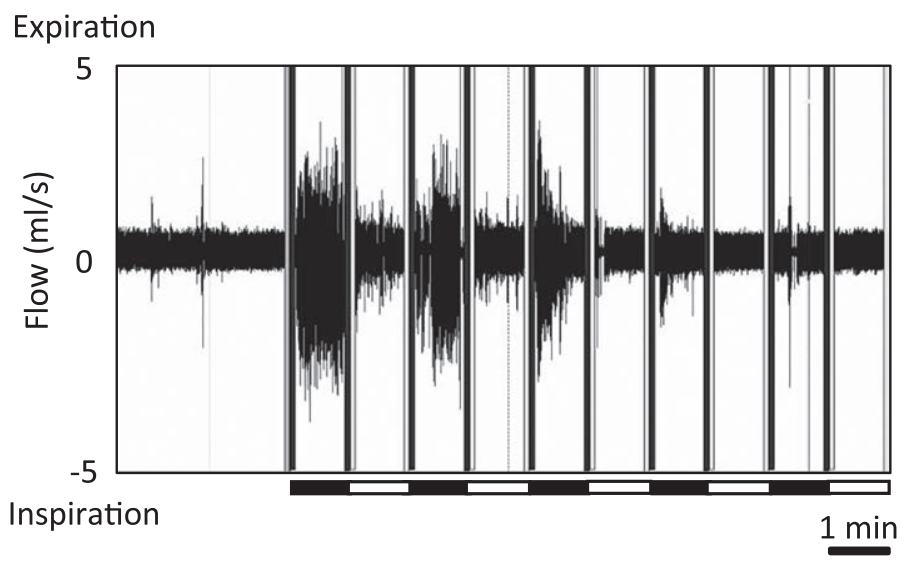

Fig. 2. Respiratory curve during photostimulation

Periods between the black axial bars show each 1 min of blue light illumination (one 500-ms pulse per min). Periods during the white axial bars show each $1 \mathrm{~min}$ of yellow light illumination (one 500-ms pulse per min).

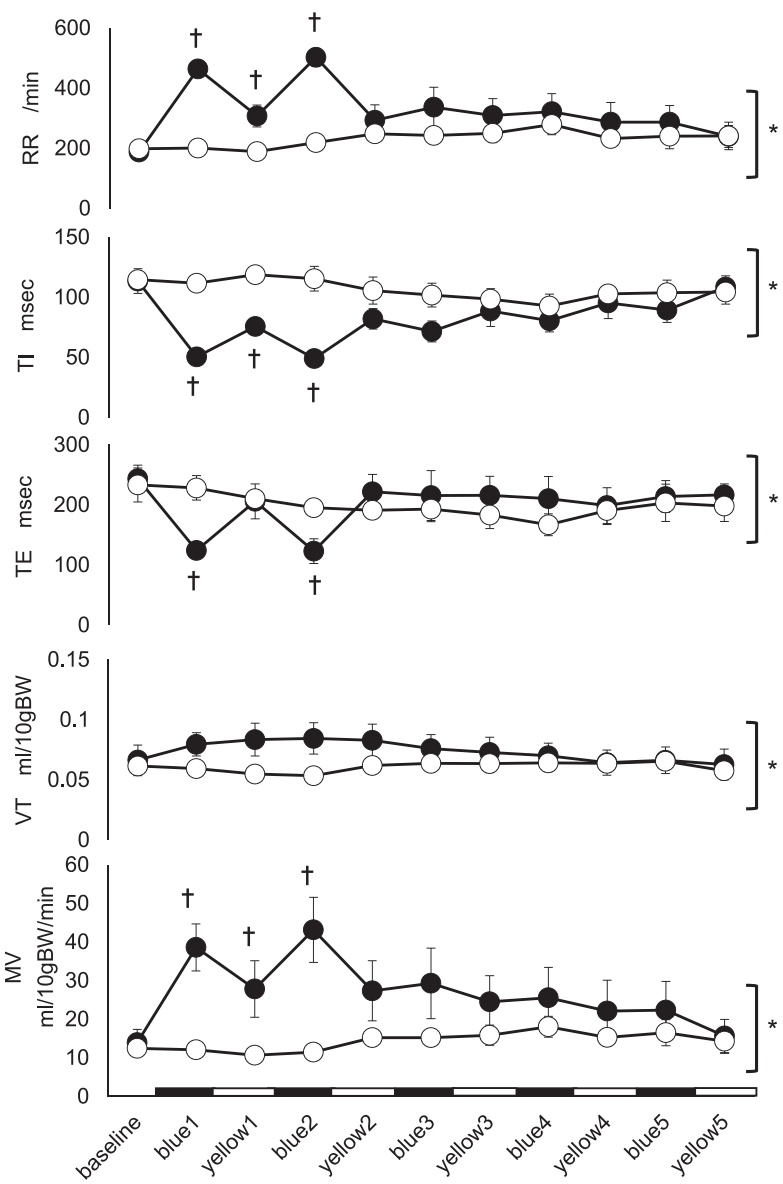

Fig. 3. Time course of changes in respiratory variables during photostimulation RR: respiratory rate, TI: inspiratory time, TE: expiratory time, VT: tidal volume, MV: minute ventilation. Data are mean \pm standard error. Open circles indicate the monogenic group $(n=6)$. Closed circles indicate the bigenic group $(n=6)$.

* : significant interaction between genotype and photostimulation

$\dagger$ : significant differences at specific times relative to baseline values 

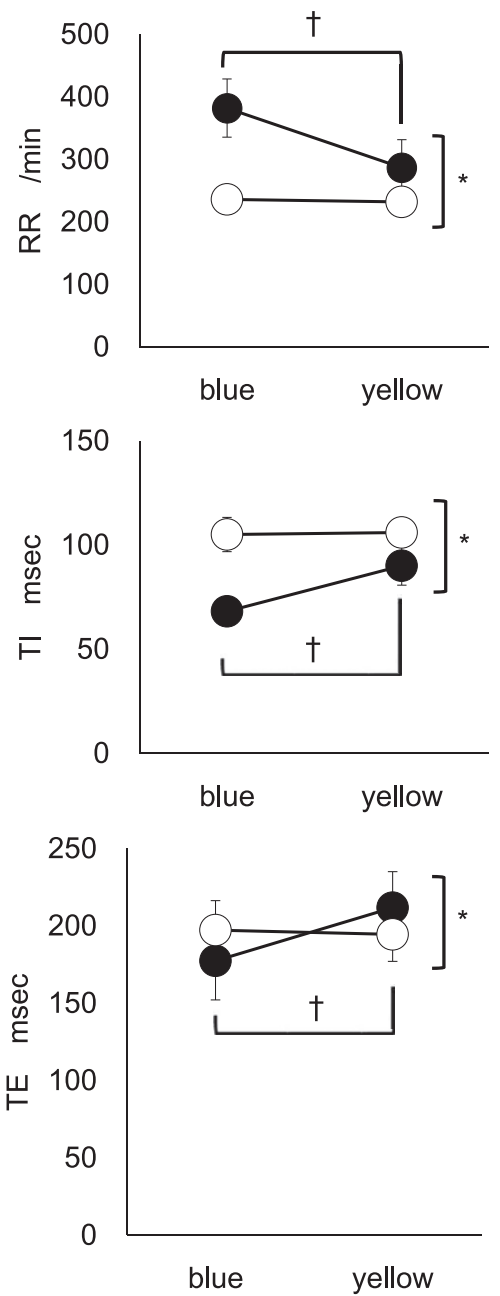
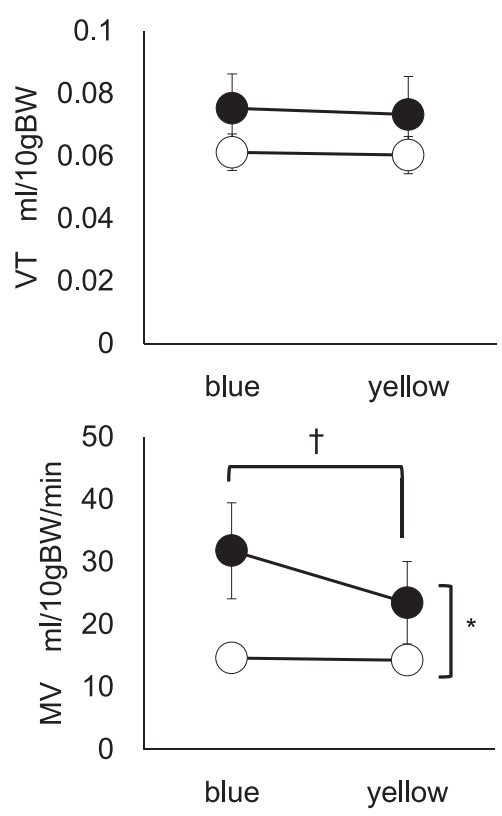

Fig. 4. Effects of blue light and yellow light illumination on respiratory variables

RR: respiratory rate, TI: inspiratory time, TE: expiratory time, VT: tidal volume, MV: minute ventilation.

Data are mean \pm standard error. Open circles indicate the monogenic group $(n=6)$. Closed circles indicate the bigenic group $(n=6)$.

* : significant interaction between genotype and photostimulation

$\dagger$ : significant differences between blue light and yellow light stimulation

a

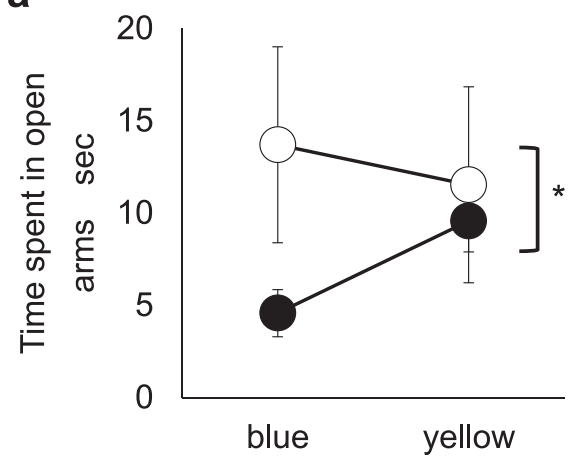

b

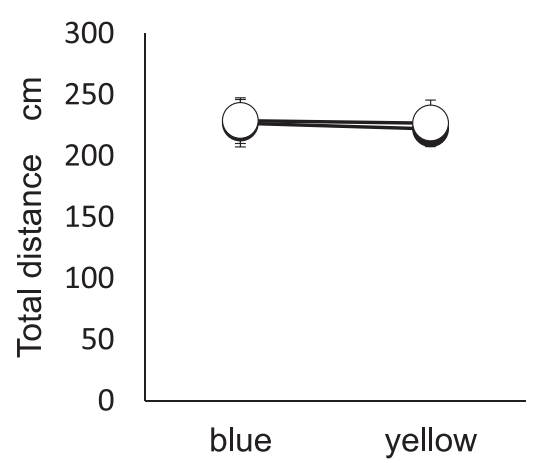

Fig. 5. Changes in time spent in the open arms of the elevated plus maze and the total distance traveled with photostimulation

a: Changes in time spent in the open arms.

b: Total distance traveled. Data are mean \pm standard error. Open circles indicate the monogenic group $(n=5)$. Closed circles indicate the bigenic group $(n=5)$.

*: significant interaction between genotype and photostimulation 


\section{Bigenic group}

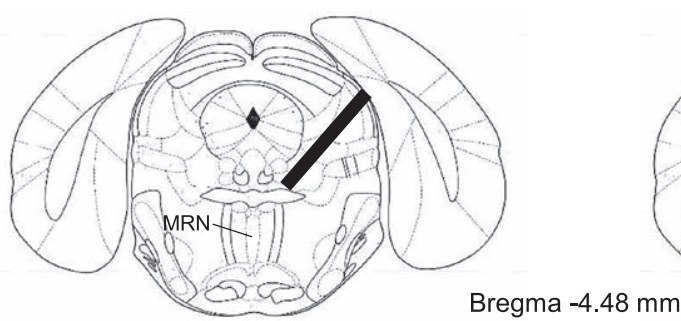

Monogenic group
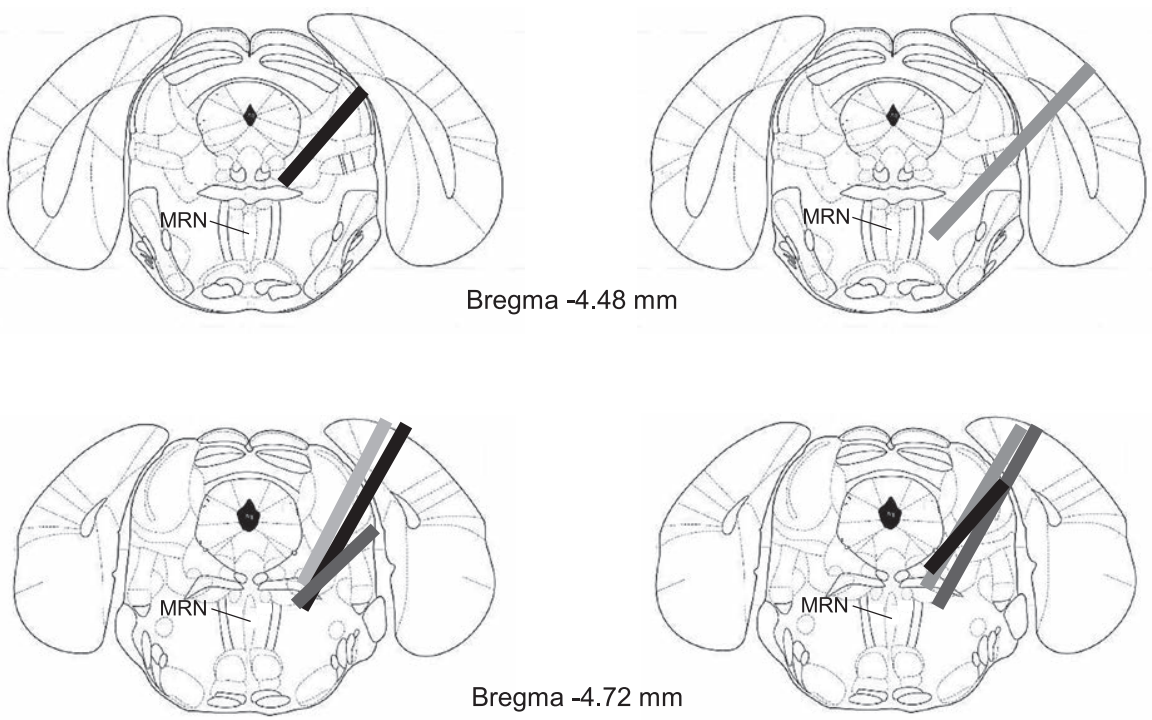

Bregma $-4.72 \mathrm{~mm}$
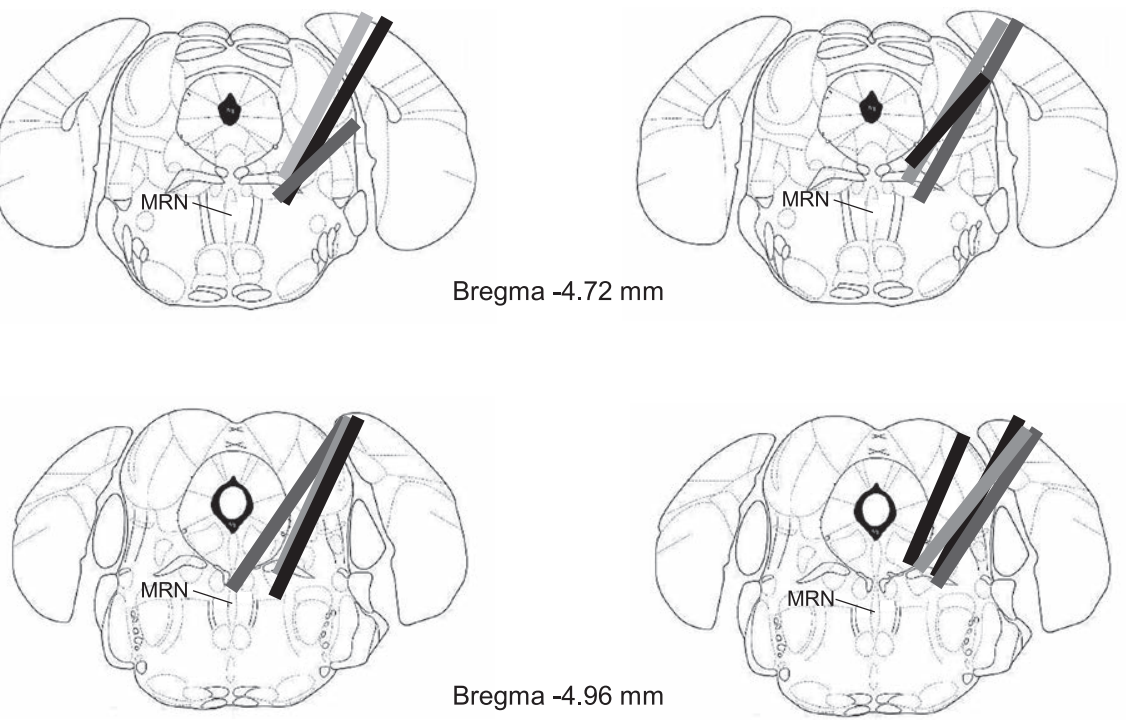

$1 \mathrm{~mm}$

Fig. 6. Locations of the optical fibers in the photostimulated MRN

The coronal sections of the brain were cut according to the mouse brain atlas ${ }^{19)}$. Bigenic group $(n=7)$ : the respiratory measurement and the following elevated plus maze test were performed in 4 of 7 mice. Monogenic group $(n=8)$ : the respiratory measurement and the following elevated plus maze test were performed in 3 of 8 mice. In both groups, either the respiratory measurement or the elevated plus maze test was performed in residual mice.

MRN: median raphe nucleus

\section{Location of optical fibers}

The optical fibers were distributed diagonally above the MRN at locations known to stimulate the 5-HT neurons in the MRN (Fig. 6) ${ }^{19)}$. The illumination was spread from the tip of each optical fiber at an angle of $60^{\circ}$. There was a similar distribution of optical fibers between the bigenic and monogenic groups.

\section{Discussion}

We confirmed that yellow fluorescence (ChR2 [C128S]-EYFP) was distributed in the DRN and MRN of the mesopontine using cleared brain blocks, and confirmed the distribution and colocalization of ChR2 [C128S]-EYFP and Tph2 in the raphe nuclei of the mesopontine. These 
findings suggest that ChR2 [C128S]-EYFP was expressed in 5-HT neurons distributed in the DRN and MRN, consistent with a previous report ${ }^{17)}$.

Our mice have expressed higher levels of ChR2 [C128S] than that in conventional mouse lines ${ }^{16)}$. A closing time constant after blue light illumination has been $106 \pm 9 \mathrm{sec}$ for the ChR2 [C128S] mutants ${ }^{15)}$. In vitro electrophysiology has also revealed a dramatic increase in MRN neuron firing rates in response to blue light illumination, and this increase was rapidly decreased by yellow light illumination ${ }^{17)}$. In our study, the respiratory curves were immediately stimulated by blue light illumination and were rapidly decreased toward baseline by the yellow light illumination. Yellow light illumination is likely to suppress respiratory facilitation elicited by blue light illumination. Therefore, our results suggest that selective 5-HT neuron activity in the MRN could facilitate respiratory rhythms due to decreases in TI and TE, and increase MV without affecting VT.

The elevated plus maze test was developed to evaluate anxiety ${ }^{20,21)}$. The actual values of the time spent in the open arms and the total distance traveled effectively translate to anxiety and locomotor activity, respectively ${ }^{17)}$. In the present study, the time spent in the open arms was significantly different between blue and alternative yellow light illumination in the MRN, without changing the total distance traveled. These findings suggest that 5-HT neural excitation in the MRN induces anxiogenic behavior without affecting the locomotor activity, consistent with previous studies ${ }^{17,22)}$.

Taken together, our results using an optogenetic method in freely moving mice suggest that endogenous 5-HT neuron activity in the MRN increases both ventilation due to respiratory frequency and anxiety. In humans, the RR increases during fear and anxiety situations ${ }^{4)}$, while during mental stress, individual anxiety levels are negatively correlated with TE, including fluctuations in TI without changes in $\mathrm{VT}^{23)}$. Our findings in 5-HT neurons of the MRN correspond to the respiratory control observed during mental stress, such as fear and anxiety in humans.

The septo-habenular linkage is important for anxious behavior, especially the triangular septumventral medial habenula, which is the core part of the interpeduncular nucleus pathway ${ }^{24)}$. 5-HT neurons in the MRN provide a moderate-to-high innervation of anxiety-related areas, including the hippocampus, septum, medial habenula, and interpeduncular nuclei ${ }^{10)}$. Anxiety is mediated via 5 -HT2C receptors in the ventral hippocampus ${ }^{25)}$. The septo-hippocampal axis is related to integration between the respiratory and limbic pathways ${ }^{11)}$. Therefore, 5-HT neurons in the MRN can stimulate the septo-hippocampal pathway, and may elicit anxiety and respiratory control.

Microinjection of L-glutamate into the MRN of anesthetized rats in a previous study, exciting some neuronal cells in addition to 5-HT neurons, induced negligible effect on respiratory responses $^{26)}$. Our results of marked respiratory responses mediated via 5-HT neurons in the MRN thus likely reflect the advantages of optogenetics such as selective stimulation of only 5-HT neurons in freely moving mice.

In the present study, respiratory facilitation was also decreased by repeated illumination of 
blue light. Although this phenomenon is well known in electrophysiological responses ${ }^{16)}$ the mechanisms underlying these repeated responses are unclear.

Light propagation in brain tissue for in vivo optogenetics exponentially diminishes with the distance between optical fiber tip and target area ${ }^{27)}$. Responses of respiration and anxietylike behavior are therefore likely caused by illumination of the MRN rather than of the raphe magnus nucleus, which was further distanced from the optical fiber.

Respiratory responses and anxiety-like behaviors were prominent, when the optical fibers were located to the upper and near the target area, and this could reflect the large photostimulation area.

Medullary 5-HT neurons play a role in ventilatory enhancement including VT changes, and contribute to metabolic respiratory control $^{28)}$. It was recently reported that 5-HT inactivation of the rostral medullary raphe attenuates tachypnea during intruder-stress, although VT was not measured $^{29)}$; however, there are few reports about respiratory control in the MRN. Further studies are needed to understand the role of MRN 5-HT neurons in respiratory control.

\section{Conclusion}

Using an optogenetic method in freely moving mice, we have shown that 5-HT neurons in the MRN are involved in the neural control of anxiety and respiration. Specifically, this experimental system can be useful to clarify anxiety-related respiratory control mediated through the 5-HT nervous system.

\section{Acknowledgments}

This work was supported by JSPS KAKENHI Grant Number JP16K08529. We thank Mr. Tomohiko Hosono for technical support in producing the transgenic mouse lines, and Edanz Group (www.edanzediting.com/ac) and InterBiotech (www.inter-biotech.com) for editing a draft of this manuscript.

\section{Conflict of interest disclosure}

The authors have no conflicts of interest to declare.

\section{References}

1) Tobin MJ, Chadha TS, Jenouri G, et al. Breathing patterns. 2. Diseased subjects. Chest. 1983;84:286-294.

2) Masaoka Y, Homma I. Anxiety and respiratory patterns: their relationship during mental stress and physical load. Int J Psychophysiol. 1997;27:153-159.

3) Giardino ND, Friedman SD, Dager SR. Anxiety, respiration, and cerebral blood flow: implications for functional brain imaging. Compr Psychiatry. 2007;48:103-112.

4) Boiten FA, Frijda NH, Wientjes CJ. Emotions and respiratory patterns: review and critical analysis. Int J Psychophysiol. 1994;17:103-128.

5) Lucki I. The spectrum of behaviors influenced by serotonin. Biol Psychiatry. 1998;44:151-162.

6) Richerson GB. Serotonergic neurons as carbon dioxide sensors that maintain $\mathrm{pH}$ homeostasis. Nat Rev Neurosci. 2004;5:449-461.

7) Harada T, Sakamoto K, Ishigooka J. Incidence and predictors of activation syndrome induced by antidepressants. 
Depress Anxiety. 2008;25:1014-1019.

8) Andrade TG, Graeff FG. Effect of electrolytic and neurotoxic lesions of the median raphe nucleus on anxiety and stress. Pharmacol Biochem Behav. 2001;70:1-14.

9) De Almeida RM, Giovenardi M, Charchat $\mathrm{H}$, et al. 8-OH-DPAT in the median raphe nucleus decreases while in the medial septal area it may increase anxiety in female rats. Neurosci Biobehav Rev. 1998;23:259-264.

10) Muzerelle A, Scotto-Lomassese S, Bernard JF, et al. Conditional anterograde tracing reveals distinct targeting of individual serotonin cell groups (B5-B9) to the forebrain and brainstem. Brain Struct Funct. 2016;221:535561. (accessed 2014 Nov 18) Available from: https://www.ncbi.nlm.nih.gov/pmc/articles/PMC4750555/pdf/429_2014_ Article_924.pdf

11) Tsanov M, Chah E, Reilly R, et al. Respiratory cycle entrainment of septal neurons mediates the fast coupling of sniffing rate and hippocampal theta rhythm. Eur J Neurosci. 2014;39:957-974.

12) Zhang F, Gradinaru V, Adamantidis AR, et al. Optogenetic interrogation of neural circuits: technology for probing mammalian brain structures. Nat Protoc. 2010;5:439-456.

13) Jiang J, Cui H, Rahmouni K. Optogenetics and pharmacogenetics: principles and applications. Am J Physiol Regul Integr Comp Physiol. 2017;313:R633-R645.

14) Nagel G, Szellas T, Huhn W, et al. Channelrhodopsin-2, a directly light-gated cation-selective membrane channel. Proc Natl Acad Sci USA. 2003;100:13940-13945.

15) Berndt A, Yizhar O, Gunaydin LA, et al. Bi-stable neural state switches. Nat Neurosci. 2009;12:229-234.

16) Tanaka KF, Matsui K, Sasaki T, et al. Expanding the repertoire of optogenetically targeted cells with an enhanced gene expression system. Cell Rep. 2012;2:397-406.

17) Ohmura Y, Tanaka KF, Tsunematsu $\mathrm{T}$, et al. Optogenetic activation of serotonergic neurons enhances anxiety-like behaviour in mice. Int J Neuropsychopharmacol. 2014;17:1777-1783.

18) Susaki EA, Tainaka K, Perrin D, et al. Advanced CUBIC protocols for whole-brain and whole-body clearing and imaging. Nat Protoc. 2015;10:1709-1727.

19) Paxinos G, Franklin BJF. The mouse brain in stereotaxic coordinates. 2nd ed. San Diego: Academic press; 2001.

20) Pellow S, File SE. Anxiolytic and anxiogenic drug effects on exploratory activity in an elevated plus-maze: a novel test of anxiety in the rat. Pharmacol Biochem Behav. 1986;24:525-529.

21) Lister RG. The use of a plus-maze to measure anxiety in the mouse. Psychopharmacology (Berl). 1987;92:180-185.

22) Teissier A, Chemiakine A, Inbar B, et al. Activity of raphe serotonergic neurons controls emotional behaviors. Cell Rep. 2015;13:1965-1976.

23) Masaoka Y, Homma I. Expiratory time determined by individual anxiety levels in humans. J Appl Physiol (1985). 1999;86:1329-1336.

24) Yamaguchi T, Danjo T, Pastan I, et al. Distinct roles of segregated transmission of the septo-habenular pathway in anxiety and fear. Neuron. 2013;78:537-544.

25) Alves SH, Pinheiro G, Motta V, et al. Anxiogenic effects in the rat elevated plus-maze of 5-HT (2C) agonists into ventral but not dorsal hippocampus. Behav Pharmacol. 2004;15:37-43.

26) Alvarenga RM, Pires JG, Futuro Neto HA. Functional mapping of the cardiorespiratory effects of dorsal and median raphe nuclei in the rat. Braz J Med Biol Res. 2005;38:1719-1727.

27) Yizhar O, Fenno LE, Davidson TJ, et al. Optogenetics in neural systems. Neuron. 2011;71:9-34.

28) Dias MB, Nucci TB, Margatho LO, et al. Raphe magnus nucleus is involved in ventilatory but not hypothermic response to CO2. J Appl Physiol (1985). 2007;103:1780-1788.

29) Ikoma Y, Kusumoto-Yoshida I, Yamanaka A, et al. Inactivation of serotonergic neurons in the rostral medullary raphe attenuates stress-induced tachypnea and tachycardia in mice. Front Physiol. 2018;9:832. (accessed 2018 Jul 10) Available from: https://www.ncbi.nlm.nih.gov/pmc/articles/PMC6050454/pdf/fphys-09-00832.pdf

[Received June 3, 2019 : Accepted July 10, 2019] 